\title{
SCIENTIFIC REP

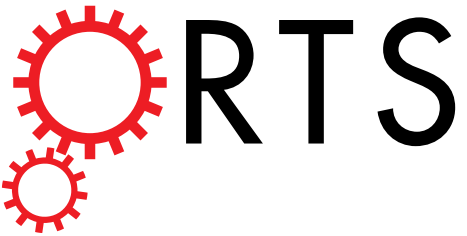 \\ OPEN \\ Author Correction: Limits of Risk Predictability in a Cascading Alternating Renewal Process Model
}

\section{Xin Lin ${ }^{1,2}$, Alaa Moussawi ${ }^{1,3}$, Gyorgy Korniss ${ }^{1,3}$, Jonathan Z. Bakdash ${ }^{4}$ \& Boleslaw K. Szymanski ${ }^{1,2}$}

Correction to: Scientific Reports https://doi.org/10.1038/s41598-017-06873-x, published online 27 July 2017

The Acknowledgments section of this Article is incomplete.

“This work was partially supported by DTRA Award No.HDTRA1-09-1-0049, by the Army Research Laboratory under Cooperative Agreement Number W911NF-09-2-0053 (the ARL Network Science Collaborative Technology Alliance). The views and conclusions contained in this document are those of the authors and should not be interpreted as representing the official policies either expressed or implied of the U.S. Army Research Laboratory or the U.S. Government".

should read:

“This work was partially supported by DTRA Award No. HDTRA1-09-1-0049, by the Army Research Laboratory under Cooperative Agreement Number W911NF-09-2-0053 (the ARL Network Science Collaborative Technology Alliance), and by the Army Research Office under grant W911NF-16-1-0524. The views and conclusions contained in this document are those of the authors and should not be interpreted as representing the official policies either expressed or implied of the U.S. Army Research Laboratory or the U.S. Government".

(i) Open Access This article is licensed under a Creative Commons Attribution 4.0 International License, which permits use, sharing, adaptation, distribution and reproduction in any medium or format, as long as you give appropriate credit to the original author(s) and the source, provide a link to the Creative Commons license, and indicate if changes were made. The images or other third party material in this article are included in the article's Creative Commons license, unless indicated otherwise in a credit line to the material. If material is not included in the article's Creative Commons license and your intended use is not permitted by statutory regulation or exceeds the permitted use, you will need to obtain permission directly from the copyright holder. To view a copy of this license, visit http://creativecommons.org/licenses/by/4.0/.

(C) The Author(s) 2018
${ }^{1}$ Social and Cognitive Networks Academic Research Center, Rensselaer Polytechnic Institute, Troy, NY, 12180, USA. ${ }^{2}$ Dept. of Computer Science, Rensselaer Polytechnic Institute, 110 8th Street, Troy, NY, 12180, USA. ${ }^{3}$ Dept. of Physics, Applied Physics and Astronomy, RPI, 110 8th Street, Troy, NY, 12180, USA. “US Army Research Laboratory, Aberdeen Proving Ground, MD, 21005, USA. Correspondence and requests for materials should be addressed to B.K.S. (email: szymab@rpi.edu) 